\title{
A Method for Classifying Cancerous and Normal Regions in Breast Thermography for Detecting Smaller Size Tumors
}

\author{
Luisa F. Polania ${ }^{1}$, Lalit K. Mestha ${ }^{1}$, Krithika Venkataramani ${ }^{2}$, L. Ramachandra ${ }^{3}$, S.S. \\ Prasad $^{3}$, Vijay Kumar ${ }^{3}$ \\ ${ }^{1}$ Palo Alto Research Center East, Webster, New York, USA \\ \{Luisa.Polania; Lalit.Mestha@parc.com $\}$ \\ ${ }^{2}$ Xerox Research Center India, Bangalore, India \\ Krithika.Venkataramani@Xerox.com \\ ${ }^{3}$ Manipal University Hospital, Manipal, India \\ \{ramachandra.l; prasad.ss; vijay.kumar@manipal.edu .
}

\begin{abstract}
Breast cancer $(\mathrm{BC})$ is one of the most frequently diagnosed cancers in women. In the United States, one in eight women are likely to be diagnosed with having some form of $\mathrm{BC}$ in her lifetime. Prevention through frequent screening can minimize the risk of mortality through $\mathrm{BC}$. The ability to obtain frequent screening in low resource settings as in India is limited for relatively large populations of women. The earlier the cancer can be detected, the more likelihood that the patient responds to treatment. Accordingly, new technologies and methodologies for the detection of cancer are increasingly needed. While mammography is the current gold standard for screening, it has low sensitivity in younger women and its harmful Xrays in frequent screening can increase the risk of cancer. Incidence rates are rising in younger women, causing a relook at thermography. We propose a sparse coding-based method to jointly learn a discriminative dictionary and an optimal linear classifier for semi-automated BC detection. Training data is built from small size image patches of normal and cancerous regions. We hypothesize that the size of the detectable tumor with the proposed method could be very small. In our method, each training sample is represented as a sparse linear combination of dictionary atoms. The resulting sparse representations are used as features for the classifier. Partial proof-of-concept is shown with $96 \%$ sensitivity for sample thermal patches of malignant and normal regions. Technique is compared to mammography correlated with sonomammography in $45 \mathrm{FNAC/biopsy} \mathrm{proven} \mathrm{cancer} \mathrm{subjects.}$
\end{abstract}

Keywords: Breast cancer screening, thermographic screening, feature extraction, tumor size, sparse coding, dictionary 